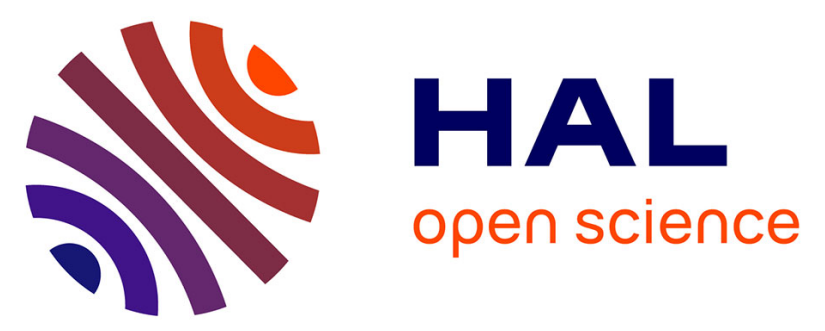

\title{
Tromperie et vérité dans trois textes brefs: Les Lais de Marie de France, La Châtelaine de Vergy, Les Miracles de Notre-Dame
}

Jean-Louis Benoit

\section{- To cite this version: \\ Jean-Louis Benoit. Tromperie et vérité dans trois textes brefs: Les Lais de Marie de France, La Châtelaine de Vergy, Les Miracles de Notre-Dame. Imposture et fiction dans les récits d'Ancien Régime, Nathalie Kremer; Jean-Paul Sermain; Yen-Maï Tran-Gervat SATOR, Jun 2013, Paris, France. pp.85-101. halshs-01415338}

\section{HAL Id: halshs-01415338 \\ https://shs.hal.science/halshs-01415338}

Submitted on 13 Dec 2016

HAL is a multi-disciplinary open access archive for the deposit and dissemination of scientific research documents, whether they are published or not. The documents may come from teaching and research institutions in France or abroad, or from public or private research centers.
L'archive ouverte pluridisciplinaire HAL, est destinée au dépôt et à la diffusion de documents scientifiques de niveau recherche, publiés ou non, émanant des établissements d'enseignement et de recherche français ou étrangers, des laboratoires publics ou privés. 


\title{
Tromperie et vérité dans trois textes brefs : Les Lais de Marie de France, La Châtelaine de Vergy, Les Miracles de Notre-Dame
}

\author{
Jean-Louis Benoit (Université de Bretagne-Sud)
}

L'imposture c'est d'abord, et essentiellement, la tromperie. Le dictionnaire Robert (édition 1980) nous rappelle cette acception première du terme «imposture »: "Action de tromper par des discours mensongers, de fausses apparences ». Il est vrai que le mot a pris aussi un sens plus précis: «tromperie de celui qui se fait passer pour ce qu'il n'est pas». Retenons de ces observations qu'il n'est pas abusif d'identifier la tromperie et l'imposture. Même si l'on fait un distinguo, la tromperie reste la mère de l'imposture. Un mari infidèle ment à son épouse. Il la trompe (dans tous les sens du mot). Par ce mensonge il joue le rôle d'un mari vertueux qu'il n'est pas. C'est un imposteur. Renart trompe son monde en faisant semblant de se convertir pour échapper à la justice. C’est un imposteur. Un marchand vend de la viande de cheval en prétendant que c'est du bœuf. Il abuse de la confiance de ses clients. Quelle imposture! Vous calomniez des gens par des accusations mensongères. Vous les faites passer pour ce qu'ils ne sont pas et vous vous passez pour un défenseur de la vérité et de la justice. Double imposture! Ne multiplions pas les exemples. Tromperie et imposture ne sont pas des concepts mais des notions très proches l'une de l'autre, au point de pouvoir établir presque toujours des liens étroits entre elles. En somme, sans prétendre les confondre totalement, en centrant notre étude sur la tromperie, nous pensons éclairer bien des formes de l'imposture tant les liens sont inextricables entre les deux termes.

Les Lais de Marie de France sont un recueil de contes folkloriques que Marie a entendu chanter en anglais et en breton. Elle s'emploie à les traduire en anglo-normand et à les versifier à la cour du roi Henri II Plantagenet, sans doute vers 1170. La Chattelaine de Vergy est un court récit de 958 octosyllabes, anonyme, qui a été écrit vers le milieu du XIII ${ }^{\mathrm{e}}$ siècle. On le considère aujourd'hui comme une nouvelle courtoise. Les Miracles de Notre Dame en français apparaissent d'abord en Angleterre sous la plume d'Adgar qui traduit et versifie les collections latines, vers 1165 (Le Gracial). Ce genre se répand partout sur le continent et atteint son apogée avec l'œuvre d'un moine du Soissonais, Gautier de Coinci, qui écrit ses Miracles de Nostre-Dame entre 1218 et 1233. Là encore, il s'agit d'un recueil de contes pieux, d'histoires, où la Vierge intervient pour sauver ou aider, d'une manière ou d'une autre, un de ses dévots. Ce sont, le plus souvent, des êtres ordinaires, des pécheurs, clercs ou laïcs. Ces miracles sont situés partout dans le monde et à toutes les époques. Chaque récit est accompagné d'un commentaire de l'auteur. Le 
seul point commun de ces œuvres, hormis la proximité des dates, est leur brièveté. En ce qui concerne les Lais et les Miracles, si chacun des récits est relativement bref l'ensemble forme un recueil imposant : 30000 vers environ pour les Miracles de Nostre Dame de Gautier de Coinci. La question de la tromperie est récurrente dans ces œuvres. On peut parler de motif littéraire qui se décline en différents topoï.

Tromper, nous disent les dictionnaires, c'est induire en erreur par divers moyens: le mensonge, la dissimulation, la ruse. Tromper est évidemment immoral, car c'est aller à l'encontre d'une loi morale universelle : il faut dire la vérité, il ne faut pas mentir. Cette loi, gravée sur des tables de pierre, souffre pourtant des exceptions. On peut tromper une personne mal intentionnée, pour l'empêcher de nuire. On peut tromper quelqu'un pour son bien. Il y a une casuistique de la tromperie et du mensonge. On sait que la ruse est loin d'avoir toujours une connotation négative, surtout dans la littérature médiévale où les rusés font souvent l'admiration et pas seulement dans le Roman de Renart. Et puis on peut se tromper soi-même, en même temps que l'on trompe autrui. C'est une faiblesse humaine excusée par toutes les sagesses du monde.

Nous examinerons les formes multiples de la tromperie dans ces textes, pour les rattacher au problème de la communication (mentir c'est toujours altérer le langage) qui est à l'arrière-plan de toutes ces tromperies. Nous nous demanderons quelle est leur fonction et pour terminer nous poserons le problème, plus large, de la vérité et de la fiction de ces œuvres tel que le posent les auteurs, reliant ainsi la problématique des personnages à celle des écrivains.

Le mensonge est omniprésent dans les Lais de Marie de France. La narratrice elle-même en est victime. Dans le prologue de Guigemar qui est, sans doute, antérieur au prologue général, Marie affirme avec fierté sa place parmi les écrivains. Elle confie qu'elle souffre des calomnies par lesquelles on cherche à la rabaisser et à la dénigrer :

Oëz Seignur, que dit Marie,

$\mathrm{Ki}$ en sun tens pas ne s'oblie.

Celui deivent la genz loër,

Ki en bien fait de sei parler.

Mais quant il a en un païs

Hume ne femme de grant pris,

Cil ki de sun bien unt envie

Sevent en dient vileinie. (Guigemar, v. 3-10 ${ }^{1}$ )

( «Écoutez donc, seigneurs, les récits de Marie qui tient sa place parmi les auteurs de son temps. On doit faire l'éloge de celui qui a une bonne réputation. Pourtant, quand un pays

\footnotetext{
${ }^{1}$ De France Marie, Lais, éd. WARnKe Karl, trad. Harf-LANCNER Laurence, Paris, Librairie Générale Française, «Le livre de poche », 1990, p. 27.
} 
possède un homme ou une femme de grand mérite, les envieux se répandent en calomnies pour diminuer sa gloire. »)

La cour, qui est le milieu dans lequel vit et écrit Marie, comme l'indique la dédicace de son œuvre au roi Henri II, est un milieu impitoyable où l'on médit des uns et des autres par jalousie. Il en va de même dans le cadre de la diégèse pour les personnages. Le lai de Lanval nous présente la cour la plus prestigieuse qui soit, celle du roi Arthur. Là aussi, c'est la jalousie et l'hypocrisie qui règnent. Lanval qui est pourtant de noble naissance, est mis à l'écart. On lui adresse des fausses marques d'amitié (p. 135, v. 24-26), on souhaite son malheur. La pire des calomnies sera le fait de la dame courtoise par excellence, Guenièvre, l'épouse du roi, dépitée par le refus de Lanval, qui l'a éconduite quand elle lui a avoué son amour. Elle commence par l'accuser de ne s'intéresser qu'aux garçons, puis, accusation plus grave, elle se plaint au roi d'avoir été sollicitée par Lanval. D'après la reine, celui-ci, furieux de son refus, aurait prétendu avoir une amie plus belle qu'elle. La reine mêle ainsi, habilement, la vérité et le mensonge. On reconnaît le motif de la femme de Putiphar $^{2}$ qui, dans la Bible, accuse Joseph de lui avoir fait des avances alors que c'est elle qui s'est rendue coupable de ce comportement adultère. Joseph est alors emprisonné. On retrouve exactement le scénario de la séductrice calomniatrice dans la Chattelaine de Vergy ${ }^{3}$. On se situe ici aussi dans le cadre d'une cour princière prestigieuse, parangon de la vie courtoise, la cour de Bourgogne. La duchesse de Bourgogne est tombée amoureuse d'un beau chevalier qui «aime ailleurs ", à savoir la jeune châtelaine de Vergy. Humiliée par le refus du chevalier, à qui elle propose son amour, elle n’hésite pas à l'accuser devant le duc d'avoir tenté de la séduire. Mensonge qui aura des conséquences tragiques pour tous les protagonistes. C'est toujours le cas pour les médisances et les calomnies. Elles se répandent et finissent par se retourner contre leur auteur, victime du mal qu'il a répandu. La mère de Frêne, qui avait accusé publiquement une femme ayant accouché de jumeaux, d'être la femme de deux hommes, se retrouve dans la même situation, quand elle accouche de deux jumelles et craint le déshonneur :

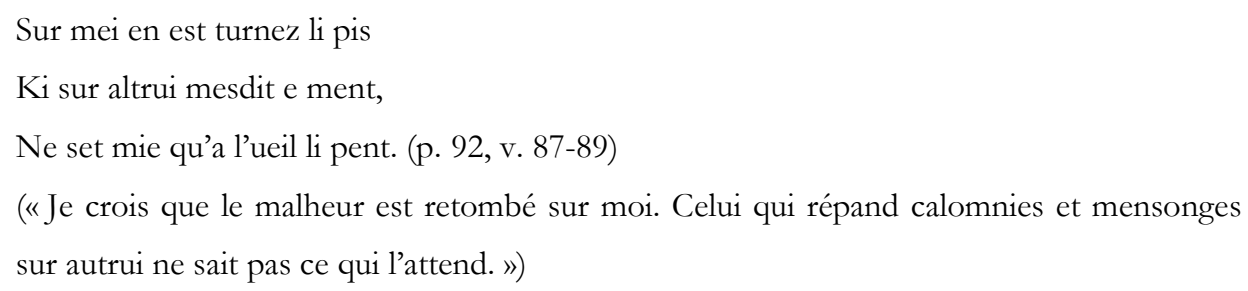

Si Marie de France déteste ce vice, c'est qu'elle a été la première à en souffrir. Elle le rattache à des défauts plus graves encore : la jalousie, l'orgueil, la haine, la malveillance. Sur ce point, comme sur bien d'autres, Marie n’hésite pas à moraliser en intervenant dans son récit.

\footnotetext{
${ }^{2}$ Genèse 39, 7-20.

${ }^{3}$ La Châtelaine de Vergy, éd. Dufournet Jean et Dulac Liliane, Paris, Gallimard, « Folio », 1994.
} 
Tromper autrui par le mensonge peut prendre bien des formes. Il en est une à laquelle on ne songe pas spontanément et qui est de règle dans les Lais : le secret. C'est là un thème fort présent dans toutes les histoires plus ou moins dépendantes de l'idéologie courtoise. On sait que c'est une règle d'or de la fine amor; c'est le seul moyen de préserver un amour menacé par les jaloux calomniateurs, les traittres qui dénoncent les parfaits amants. Taire la vérité, la cacher aux yeux des autres, c'est les tromper. Sur ce point, la tromperie est générale dans les Lais, même si elle est présentée comme positive. Le Bisclavret cache longtemps les raisons de sa disparition. La fée qui aime Lanval lui impose la sacro-sainte promesse de garder le secret:

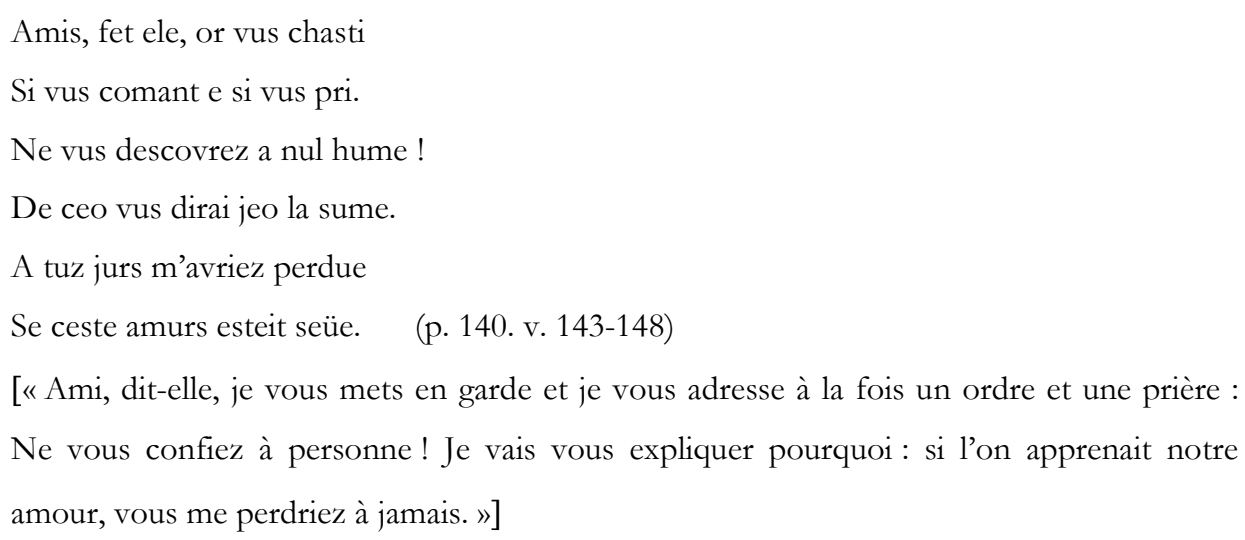

Tous les amants des Lais, ou presque, tâchent de garder leur amour secret, par toutes sortes de dissimulations et de cachotteries. La dame de Yonec rêve de cette époque bénie où les femmes pouvaient avoir des amis sans encourir de blâme, car elles étaient les seules à les voir. Elle sera exaucée et elle entretiendra longtemps une relation avec son bel oiseau de chevalier. Même chose pour la dame et son galant chevalier qui se parlent la nuit d'une fenêtre à l'autre, sans pouvoir se toucher. Longtemps leurs amours vont rester secrètes, comme relaté dans Le Rossignol. Dans Le Lai du chèvrefenille, Iseut parvient à retrouver secrètement Tristan, grâce à son extrême maîtrise de la ruse et du mensonge, qui sont d'ailleurs valorisés dans tous les textes qui retracent sa légende.

On peut aussi mentir par omission. C'est même le cas, quand on veut garder un secret et l’imposer à ses proches. Éliduc, épris de la jeune Guilladon, n’avouera jamais à sa bien-aimée qu’il est marié avec Guildeluec (imposture élémentaire !). Il faut que ce soit un matelot qui le révèle à la pauvre jeune fille, lors d'une traversée périlleuse. Elle en mourra de chagrin, ou presque.

La Châtelaine de Vergy repose sur ce secret intenable d'un amour caché entre le chevalier et la châtelaine. Les deux amants vont déployer beaucoup d'efforts pour le préserver et cacher la vérité à ceux qui veulent le percer, notamment la duchesse, qui est la pire des menteuses.

Bien souvent mensonge, vérité tue, silence trompeur ont un même but: tromper au sens amoureux du terme. L'adultère n'est guère condamné par l'idéologie courtoise et chez Marie, dont la position est complexe à ce sujet, c'est souvent un amour adultère qui est valorisé. La jeune épouse du vieux mari jaloux dans Yonec trompe son mari avec la bénédiction de la narratrice. Il en 
va de même pour la dame du lai du Rossignol qui aime le chevalier de la maison voisine. Elle ne peut hélas le rejoindre, mais elle se lève toutes les nuits pour « l'aimer de loin ». Éliduc, qui a une liaison avec Guilladon, trompe à la fois sa femme et la jeune fille à laquelle il ment. Tantôt cette infidélité est condamnée, et les amants le sont aussi, tantôt elle est justifiée et les amants sont innocentés. Pour les amants coupables, citons la dame et le roi dans Equitan, justement punis pour leur tentative de crime passionnel, ou le Bisclavret. L'épouse du loup-garou qui a percé le secret de son mari va le tromper avec un chevalier en condamnant son mari à rester un loupgarou sans qu'il ne puisse jamais reprendre sa forme humaine. Elle sera elle aussi châtiée.

Pour tromper, au sens amoureux, tous les moyens sont bons. Les personnages déploient une ruse inouie. Le chevalier qui aime la dame dans Yonec se métamorphose en oiseau, mais bien plus encore, il ira jusqu’à se métamorphoser en la dame pour recevoir l'hostie afin de prouver, de manière bien peu convaincante à nos yeux ${ }^{4}$, qu'il est une créature divine et non pas diabolique. On sait bien que le Diable est le père du mensonge. Il ne faut pas s'étonner que les maris jaloux usent des mêmes armes que leurs épouses volages et leurs rivaux. Eux aussi tendent des pièges, au sens propre du mot, quand il s'agit de blesser le chevalier oiseau qui s'introduit par la fenêtre pour s'unir à sa dame. Il en va de même pour le mari dans le conte Le Rossignol. Il ne dit pas à son épouse qu'il a compris son stratagème, qui consiste à lui faire croire qu'elle se lève la nuit pour écouter le chant du rossignol. Il se contente de piéger le pauvre animal, de le tuer et de le jeter sans rien dire sur son épouse, comme un symbole de sa victoire. Mensonges cruels et mensonges enjolivés de grâce courtoise se répondent dans un dialogue faussé.

Dans ce monde de la courtoisie, si peu transparent, si menaçant qu'il faut se cacher ou mentir pour survivre ou pour aimer, la parole est constamment empêchée, interdite. Communiquer est le problème majeur des personnages. La seule parole vraie n'est possible que de manière détournée. Partout, ce sont des messages secrets, des codes, des signes qui viennent suppléer aux interdits que subissent les amants. Le chevalier et la châtelaine de Vergy savent se servir d'un petit chien qui fait signe au chevalier qu'il peut retrouver sa maittresse. Un cygne, cet oiseau mythique, sera le messager de Milon et de sa dame. Des objets signaleront l'identité d'enfants cachés et abandonnés ou la complicité des amants (chemise et ceinture de Guigemar, étoffe et anneau de Frêne).

\footnotetext{
${ }^{4}$ Cf. BenOit Jean-Louis, «Yonec, une nouvelle vengeance du fils de la veuve ?», in FerLAmpIn-Acher Christine et HuË Denis, Lignes et lignages, dans la littérature arthurienne, Presses Universitaires de Rennes, 2007, p. 153-165.
} 
Tristan et Iseut restent ici encore les maitres de la communication impossible. Le message mystérieux gravé sur le bâton de coudrier, qui a fait couler beaucoup d'encre ${ }^{5}$, suffit à attirer l'attention vigilante d'Iseut. L'amour rend intelligent, sensible, inventif. Lui seul permet de percer le sens des signes les plus discrets que s'adressent les amants. Lui seul rend poète, car la poésie est le langage réservé à ceux qui s'aiment et qui peuvent ainsi dire l'indicible, la vérité invisible au vulgaire. Le mari jaloux, qui a tué le rossignol qui unissait les amants, n'a pas gagné la partie par son geste cruel et cynique. En mettant le corps du pauvre animal dans un coffre précieux, le chevalier offre à sa dame un signe définitif d'un amour éternel. Il en fait une relique sacrée. Bien plus, en enveloppant le corps de l'oiseau d'une étoffe précieuse sur laquelle est écrite leur histoire, il célèbre cet amour tragique avec l'éclat de la poésie, seul moyen d'échapper à la violence et à l'absence de communication. Les amants veulent se parler. Iseut et Tristan, qui se retrouvent grâce à leur "bâton poème », ne font pas autre chose et leur passion intacte se libère en un échange enfin libre de propos :

Entre els meinent joie mult grant

A li parla tut a leisir

E ele li dist sun plaisir. (Le Chèvrefenille, p. 266, v. 94-96)

[«Ils ont enfin la joie de se retrouver ! Il peut lui parler à son aise et elle lui dire tout ce qu'elle veut. »]

Dans ces récits brefs, si proches des contes folkloriques pour Marie de France, il ne faut pas exagérer l'aspect psychologique des motivations des personnages. Mensonges, secrets, trahisons, dénonciations, calomnies, etc.: toutes ces formes de la tromperie (facteurs de diverses impostures) jouent surtout un rôle fonctionnel dans la narration. Aussi, ce que Propp affirme à propos des personnages des contes est en grande partie valable pour ceux des Lais:

La volonté des personnages, leurs intentions ne peuvent être tenues pour des signes consistants... Ce n'est pas ce qu'ils veulent faire qui est important, ce ne sont pas les sentiments qui les animent, mais leurs actes en tant que tels, définis et évalués, du point de vue de leur signification pour le héros et pour le déroulement de l'intrigue 6 .

Le schéma narratif déroule dans ces formes brèves ses épisodes convenus. L'élément perturbateur est très souvent constitué par la tromperie exercée par un opposant au héros. Les péripéties qui en découlent semblent ôter toute liberté à ces personnages qui sont des types fonctionnels. D’ailleurs prophéties et prédictions viennent jalonner la diégèse pour mieux marquer le caractère inéluctable de la destinée du héros. Dans ces histoires où le schéma des actants est réduit à son expression la plus simple, il est évident, comme le note Metelinski, que

\footnotetext{
${ }^{5}$ Cf. TrachsLer Richard, Tant de lettres sur un si petit bastun, Le Lai du Chèvrefeuille devant la critique littéraire 1200-2000, Salerno éditrice, 2003.

${ }^{6}$ Propp Vladimir, Morphologie du conte, Paris, Seuil, 1970, p. 30.
} 
«chaque injonction, chaque prescription ou recommandation doit être exécutée... Par contre, chaque interdiction doit être violée, stimulant ainsi également une action ${ }^{7}$. »

Dans les lais, il en va ainsi, les secrets sont faits pour être percés, les amants pour être trahis, les héros pour être trompés, puis détrompés. Ce sont là des facteurs d'une intrigue qui a besoin de ces rebondissements pour arriver à la vérité ultime de la situation finale qui n’est pas toujours positive chez Marie. Notons que le merveilleux est un moyen de lever les obstacles et de permettre au héros de parvenir à une issue heureuse. L'exemple le plus évident est celui de Lanval. Le héros, victime d'une machination implacable parvient à se justifier et à échapper à un injuste châtiment grâce à l'intervention de sa fée bien-aimée qui vient illuminer la cour d'Arthur de sa beauté et emmener son amant à Avalon.

Il n'en va pas de même dans La Châtelaine de Vergy où la situation, faite d'un enchevêtrement de mensonges, de secrets trahis et de malentendus en cascade ne peut échapper à une logique tragique, car ici, rien ne vient arracher les héros à la malveillance des méchants et aux contradictions de l'amour courtois ${ }^{8}$. Alors la tromperie est assassine, ce qui différencie les lais merveilleux de la nouvelle courtoise. L'autre différence est la profondeur psychologique attribuée aux personnages de la nouvelle. Ils aiment, souffrent, se déchirent et se lamentent dans les affres de la fatalité, avec une vraisemblance et une intensité que n’ont pas toujours les héros des lais. La tromperie volontaire (celle de la duchesse), forcée (celle du chevalier), subie et imaginaire (celle de la châtelaine qui croit à l'infidélité de son amant) est un facteur incontestable de pathétique, dans un univers marqué, lui aussi, par l'incompréhension et l'incommunicabilité entre les êtres.

Nous avons laissé de côté, jusqu'à présent, Les Miracles de Notre Dame, comparables, en de nombreux points aux œuvres précédentes, par leur brièveté, le schématisme des personnages et les enjeux narratifs. Cependant une différence change tout, c'est la présence de Dieu qui vient régler les problèmes par l'intermédiaire de la Vierge. Nous ne ferons pas un fastidieux relevé des modalités de la tromperie et du mensonge dans les miracles. Nous nous contenterons de deux messages édifiants. Parmi le panorama exhaustif et effrayant des péchés des hommes que présentent les textes, il en est un qui, aux yeux des auteurs ecclésiastiques, est gravissime. Il s'agit de l'infidélité d'un clerc ou d'une religieuse à ses vœux. Séduit par une jeune fille, courtisée par un chevalier, le jeune clerc ou la religieuse quitte la vie monastique. Il s'agit alors d'une infidélité où la victime n'est pas un homme ou une femme, mais c'est Dieu lui-même qui est trompé. Citons chez Adgar, dans le Gracial, les miracles suivants (on trouve à peu près les mêmes avec des

\footnotetext{
${ }^{7}$ Metelinski Eleazar, « Problème de la morphologie historique du conte populaire », Semiotika, n 2, 1970, p. 133.

${ }^{8}$ Cf. Maraud André, «Le lai de Lanval et La Chastelaine de Vergi: la structure narrative », Romania, n 93, 1972, p. 456. Sur cet enchaînement fatal de secrets trahis, voir Dufournet Jean et Dulac Liliane, La Chastelaine de Vergy, Paris, Gallimard, "Folio », 1994, p. 160.
} 
variantes chez Gautier de Coinci): le sacristain noyé (II), le clerc de Chartres (III), le clerc amoureux (XXXVI), la sacristine (XLVIII) ${ }^{9}$.

Les infidélités sont chaque fois réparées par la Vierge qui inspire au pécheur le repentir salutaire, parce que celui-ci a gardé une dévotion pour elle. Parfois, elle intervient même comme une femme jalouse qui vient reprocher à un ami de l'avoir délaissé. Ce type de conte est appelé «le fiancé de la Vierge».

Il est un type de miracles où la tromperie est l'œuvre de celui qui en est le maittre absolu, l'usurpateur, l'Imposteur par excellence (Ne cherche-t-il pas à se faire passer pour Dieu ?), le Diable. Il est capable de se déguiser pour abuser et capturer le pécheur. L'ange des ténèbres devient faux ange de lumière. Ainsi, le pauvre pèlerin qui allait à Saint-Jacques croit avoir affaire à une apparition de saint Jacques ${ }^{10}$. Le faux saint Jacques, qui n'est autre que le démon, accable le pèlerin qui a commis un péché avec une femme. Il le persuade de se castrer pour faire pénitence. Le pèlerin s'exécute et meurt. Heureusement, au Ciel, le vrai saint Jacques viendra plaider sa cause et le ressuscitera, légèrement diminué. Il entrera dans les ordres à Cluny. Chez Gautier de Coinci on trouve un miracle où le Diable prend le corps d'un jeune homme pour abuser un riche seigneur :
Annemis, qui se transfigure
En forme d'angele et prend figure
Quequ'il plaist assez sovent
Le cors d'un vallet de jovent
De novel mort par guille a pris (t. 3, p. 109, v. 55-59).
[«L'Ennemi qui se transforme en ange et prend forme humaine quand il lui plait assez souvent, a pris, par ruse, le corps d'un jeune homme récemment décédé. »]

Il faudra toute la perspicacité d'un saint évêque et l'efficacité d'une prière à Marie pour que la victime échappe aux griffes de l'Ennemi et que celui-ci soit démasqué.

S’il le faut, pour sauver un de ses enfants en danger, fût-il un pécheur, la Vierge peut aussi se livrer à des déguisements ou à des métamorphoses. Un exemple frappant nous est donné par le miracle célèbre qu'on rencontre dans toutes les collections de miracles, celui dit de «la sacristine ». Une religieuse finit par céder aux avances d'un chevalier et quitte son couvent. Cette fuite n'est pas d'abord sans difficultés, car la statue de la Vierge, qu'elle avait l'habitude de saluer, lui barre plusieurs fois la porte de la sortie. Cependant, lors d'une ultime tentative, elle parvient à sortir, sans la saluer. Elle vit pendant plusieurs années avec son mari, jusqu’à ce que celui-ci soit condamné à mort pour ses crimes. Elle se repent d'avoir quitté les ordres et décide de regagner

\footnotetext{
${ }^{9}$ Adgar, Le Gracial, éd. Kunstmann P., Presses de l'Université d’Ottawa, 1982.

${ }^{10}$ Ibid., VIII. Chez De CoInCI Gautier, Les Miracles de Nostre Dame, éd. KoenIG F., 1966-1970, Genève, Droz, De celui qui se tua par l'amonestement dou dyable, t. II, p. 237.
} 
son couvent. Quelle n'est pas sa surprise de constater que personne ne s'est aperçu de sa disparition! Elle se rend compte que la Sainte Vierge a pris son apparence et l'a remplacée dans le chœur, pendant plusieurs années. On a là un exemple incontestable d'imposture au service d'une bonne cause : protéger une pécheresse. L'illusion déceptive qui fait tant de tort aux hommes dans ce monde trouble et trompeur peut donc aussi être mise au service du bien.

La relation de l'auteur au lecteur reproduit cette dialectique de la tromperie et de la vérité, présente dans les récits entre les personnages.

Les contes que je sai verais

Dunt li Bretun unt fait les lais

Vos conterai assez briefment. (Guigemar, v. 19-11, p. 26)

[ "Je vais vous raconter, en peu de mots, les contes dont je sais qu'ils sont vrais, les contes

d'où les Bretons ont tiré leurs lais »]

Ainsi Marie, dans le prologue du lai Guigemar, affirme sa volonté de raconter des histoires vraies. Bien des auteurs du Moyen Âge proclament ainsi leur volonté de dire la vérité. Pour Marie, comme pour d'autres, le critère de la vérité est d'abord la conformité à la source. Ellemême puise aux lais contés, puis chantés, par les Bretons. Contrairement à ce qu'on dit parfois, elle se veut fidèle à des sources écrites. Elle le dit explicitement dans ce même prologue de Guigemar :

El chief de cest comencement

Sulunc la letre e l'escriture

Vos mostrerai une aventure

Ki en Bretaigne la menur

Avint a tens anciennur (v. 22-26, p. 26).

[«Au terme de ce prologue, conformément au texte écrit, voici une aventure survenue, il y

a bien longtemps, en petite Bretagne. »]

Elle puise aussi, bien sûr, à des sources orales, contrairement à tant d'auteurs qui se contentent de traduire du latin en français, elle estime nécessaire de faire remembrance, de garder le souvenir des lais qu'elle a entendu chanter en anglais et en breton et qui, eux-mêmes, gardent le souvenir des aventures que les poètes avaient entendues. La vérité repose donc sur la fidélité dans la tradition, la transmission des évènements racontés et chantés. L'écrivain est un chaînon ultime au bout de cette chaîne. À l'origine, si cela est possible, on trouverait un protagoniste de l'histoire. C'est le cas idéal qui se présente pour Le Lai du Chèvrefenille, cette version exclusive d'un épisode heureux de la légende de Tristan et Yseut. En effet, Marie affirme que c'est Tristan lui-même qui aurait composé la première version du lai.

Pourtant cette vérité factuelle des aventures racontées n'est pas la plus importante. Marie ne croyait évidemment pas à ces histoires peuplées de fées et de loups-garous. La vérité est d'un 
autre ordre, elle résulte d'une relecture des textes et des chansons. Seul l'écrivain par son interprétation, par la glose qu'il peut en faire dégagera une signification plus profonde. Son prologue général explique qu'il s'agit de gloser la letre et de lur sen le surplus metre (" de commenter le texte et d'y rajouter le surplus de science qu'ils auraient »). Ainsi, à partir de légendes puisées dans un fond immémorial (qu'il soit antique ou breton), on peut transmettre un enseignement profond en matière de chevalerie, d'amour, de morale. La «matière » initiale reste irremplaçable. Il faut la conserver, la traduire, la commenter. À travers la fiction, donc le mensonge, se dégage une vérité pour les lecteurs d'aujourd'hui et de demain. Le lecteur est invité à une forme particulière de la tromperie, qui est l'illusion narrative. On fait semblant de croire une histoire qui est peut-être fausse, mais qui porte une vérité que l'auteur aide à découvrir.

Les auteurs de miracles sont plus sévères avec la littérature profane et plus exigeants avec la notion de vérité. Adgar écrit à la même date et dans le même contexte culturel que Marie, à la cour d'Angleterre. Il reconnaît à cette littérature des vertus de courtoisie et de sagesse, mais il invite à préférer les « écrits de Notre Reine », c'est-à-dire les miracles qu’il écrit :

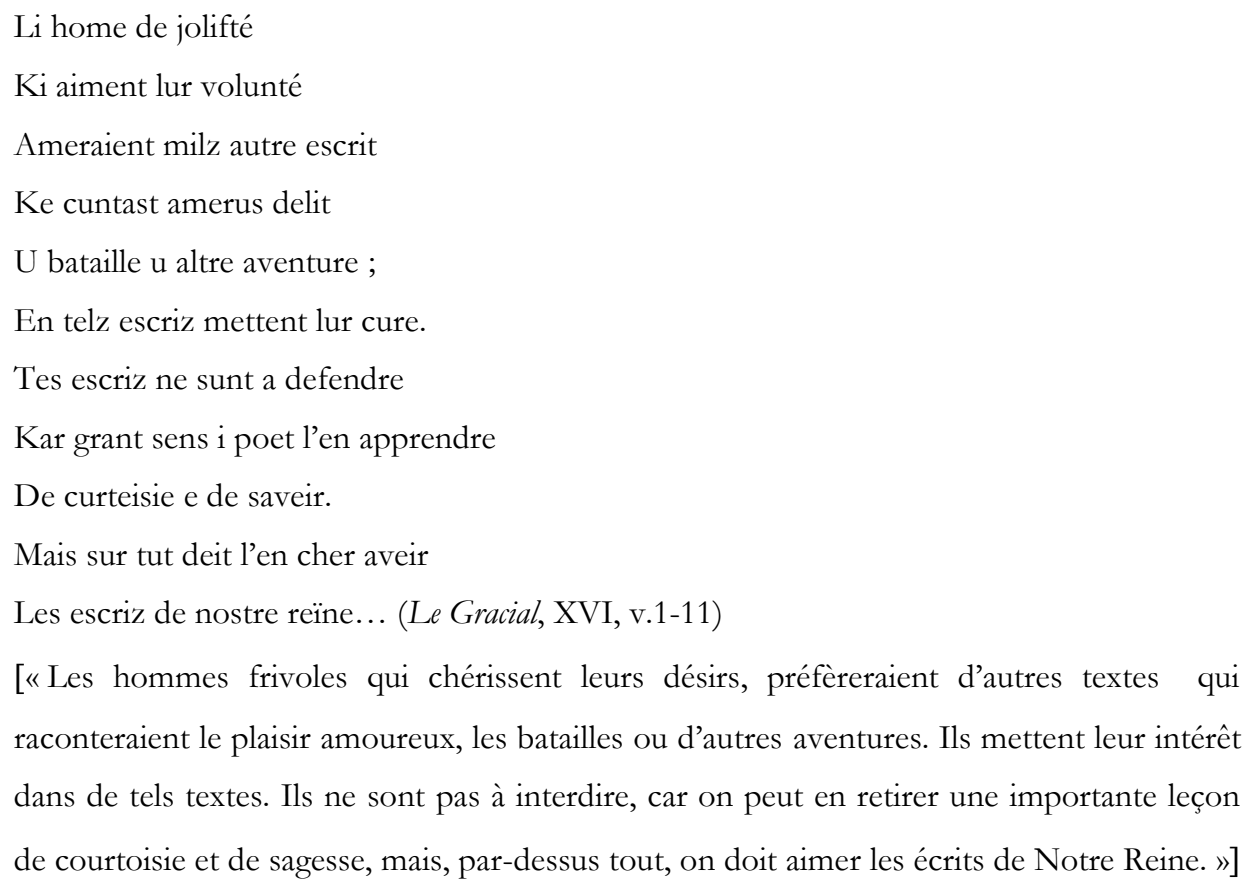

Gautier de Coinci n'a pas cette largeur de vue ni cette indulgence envers la littérature courtoise. Il la condamne sans appel :

Li menteür en enfer boutent

Et auz et cialz qui les escoutent (Prologue t. 3, p. 267)

[«Les menteurs jettent en enfer eux-mêmes et ceux qui les écoutent »].

Il n'a de cesse de condamner la littérature courtoise en général, la lyrique amoureuse, le merveilleux païen, les contes à rire (les fabliaux), le Roman de Renart et même les chansons de geste. Outre leur caractère mensonger, ces textes véhiculent un message immoral, en 
contradiction avec l'enseignement de l'Église, en matière de mariage et d'amour en particulier. Contre ces influences pernicieuses, les auteurs de miracles vont raconter des histoires vraies où Marie intervient de manière spectaculaire pour sauver des pécheurs et récompenser ses dévots. Cette littérature édifiante a le mérite d'être vraie et de fortifier la foi. Cela, sans ennuyer le lecteur, car il faut lui plaire et le séduire par un merveilleux digne de rivaliser avec le merveilleux antique ou breton, un pathétique comparable à celui des meilleurs romans d'aventures et un lyrisme marial plus émouvant que le lyrisme courtois.

Les auteurs de miracles ne sont pas des écrivains naïfs et crédules, prêts à accepter tous les miracles qu'on peut raconter. Ils cherchent des témoins dignes de foi, des autorités qui les ont transmis. Même discernement nécessaire pour les reliques dont ils font grand cas : le soulier de la Vierge à Soissons, les restes de sainte Léocadie à Vic-sur-Aisne, les cheveux de la Vierge à Laon, la sainte chemise de la Vierge à Chartres, etc. Autant ils sont attachés au culte lié à ces vraies reliques (afin de voir le châtiment infligé au bouvier blasphémateur du saint soulier), autant ils sont intransigeants envers les colporteurs de fausses reliques comme la dent de Notre Seigneur, ou ses jours de jeûne enfermés dans une bouteille (D’une fame qui fu garie a Arras, t. 4, p. 315). Le principal critère de vérité de ces auteurs est la fidélité à la letre, à la matière, c'est-à-dire au texte source que chaque auteur de miracle a la prétention de suivre fidèlement. Ce texte source, en latin, il l'a trouvé dans la bibliothèque de son monastère (de l'abbaye de Saint Paul de Londres, pour Adgar) et il constitue à ses yeux une compilation irrécusable des miracles opérés par la Vierge partout dans le monde. Il est pourtant inévitable de mettre en question l'authenticité de miracles très anciens, transmis par des intermédiaires nombreux et lointains. Les auteurs de miracles en ont conscience, c'est pourquoi, ils encadrent leur récit par un commentaire didactique qui en dégage le sens. Ainsi, Gautier de Coinci et Adgar feront comprendre que les scènes du Tribunal de Dieu où les anges et les démons se disputent les âmes en présence de saint Pierre, muni d'une clé et de la Vierge, avocate irrésistible, doivent être interprétés comme des scènes symboliques, car on ne peut rendre compte de réalités immatérielles, comme le jugement de l'âme, que par des images :

Que ço que n'ad cors ne visage

Estre ne put mustré el munt

A ceus ki cors ne visage unt

Fors par cors de aperte semblance

Pur ço ne vei ge ci dutance (Le Gracial, VII, v. 136-140)

[«Car ce qui n'a ni corps ni visage ne peut être montré dans le monde à ceux qui ont un corps et un visage, que par le moyen d'une image expressive. C'est pourquoi, je ne vois pas ici de raison de douter »]. 
Plus d'une fois, nos auteurs s'empressent de donner un sens symbolique, voire allégorique, à tel miracle, notamment le célèbre voyage allégorique du clerc d'Europe (Adgar, Le Gracial, XV). Un moine malade voyage au Ciel sous la direction d'un ange qui lui fait visiter un champ de fleurs magnifiques. Ces fleurs signifient allégoriquement les versets des psaumes qu'il avait coutume de réciter. Le miracle a besoin de sa glose. Parfois la vision survient sous la forme d'un rêve pendant le sommeil. Il faut l'interpréter (par exemple le symbolisme du lait de la Vierge qui «signifie »sa miséricorde). Ce message divin n’en a pas moins de réalité. Sa vérité, dégagée par la glose, est plus importante qu'un évènement factuel produit dans la réalité pour une conscience éveillée.

Dans les textes brefs que nous avons choisis la tromperie règne entre les personnages et fonde une universelle imposture où tout le monde ment à tout le monde. Elle constitue le facteur majeur de l'intrigue et l'arrière-plan inquiétant d'un monde marqué par le mensonge et la difficulté à établir une communication sincère propice à la vérité. Cette obscurité, cette incompréhension, ces trahisons peuvent être tragiques. Le merveilleux, celtique ou chrétien, permet d'y remédier. La voix de l'écrivain est prise dans ces problématiques complexes. Lui aussi recherche la vérité et veut la transmettre. Il est tenu de composer avec la fiction. Or écrire une fiction c'est tromper le lecteur, lui faire croire, souvent avec insistance, que l'on raconte une histoire vraie. Le lecteur est d'ailleurs, parfois, complice de cette séduisante imposture. La littérature propose une vérité plus importante que la vérité historique ${ }^{11}$. Pour la saisir, l'écrivain doit sans cesse lire et relire, commenter et réécrire les textes antérieurs sur lesquels repose son œuvre. Il doit jouer avec la fiction, donc le mensonge, emprunter des codes esthétiques et des formes qui lui permettent de dire plus qu'il ne croit. Cela à condition de trouver le cœur et l'esprit d'un lecteur capable de le recevoir et de développer le sens du texte. Marie de France inscrit dans son œuvre la figure emblématique de cet écrivain à travers le personnage de Tristan contraint d'inventer un nouveau mode de communication par la poésie et d'échapper ainsi à la tragique incommunicabilité de ce monde. Ce langage porteur de vérité, seule Iseut, lectrice amoureuse ${ }^{12}$, saura le lire.

\footnotetext{
${ }^{11}$ Cocteau écrit que le poète est un menteur qui dit toujours la vérité.

${ }^{12}$ Lectrice amoureuse de son mystérieux message dans Le Chèvrefeuille.
} 